\title{
INVARIANCE QUANTUM GROUPS OF THE DEFORMED OSCILLATOR ALGEBRA
}

\author{
Jacqueline BERTRAND and Michèle IRAC-ASTAUD \\ Laboratoire de Physique Théorique et Mathématique \\ Université Paris VII \\ 2 place Jussieu F-75251 Paris Cedex 05, FRANCE
}

submitted to Journal of Physics A : Mathematical and General.

\begin{abstract}
A differential calculus is set up on a deformation of the oscillator algebra. It is uniquely determined by the requirement of invariance under a seven-dimensional quantum group. The quantum space and its associated differential calculus are also shown to be invariant under a nine generator quantum group containing the previous one.
\end{abstract}




\section{Introduction}

Several kinds of differential calculi have been introduced on quantum groups and quantum spaces and widely studied $([1]-[3])$. However there is one important case that deserves a special treatment because of its importance in physics, namely the case of the oscillator (or Weyl-Heisenberg) algebra. Indeed, in its usual form it represents the algebra of observables in quantum mechanics. After deformation, it is still an algebra of observables but for a different quantization.

The problem of constructing a differential calculus on such an algebra can be tackled in different ways. Here we privilege the invariance approach. Given the quantum space of variables (or observables), we first construct the set of seven element quantum

matrices preserving that space (section 2). The space of differentials is then determined in two independent ways: either by postulating the existence of a $R$-matrix (section 3 ) or by constructing directly a new invariant space (section 4). The uniqueness of the result emphasizes the power of the approach based on covariance. Finally, in section 5, the quantum group preserving simultaneously the spaces of variables and differentials is explicitly described. Moreover, a larger quantum group with nine generators is also shown to preserve the same spaces. Both groups can be endowed with a structure of Hopf algebra. 


\section{The quantum space and its invariance algebra}

The Weyl-Heisenberg algebra written in homogeneous form is the free associative algebra generated by three operators $x^{i}$ satisfying the following quadratic relations :

$$
(R)\left\{\begin{array}{l}
x^{1} x^{2}-x^{2} x^{1}-s\left(x^{3}\right)^{2}=0 \\
x^{1} x^{3}=x^{3} x^{1} \\
x^{2} x^{3}=x^{3} x^{2}
\end{array}\right.
$$

This algebra will be denoted by $\mathbb{C}<x>/ R$. It is invariant under the seven-parameter Lie subgroup $G$ of $G L(3)$ consisting of matrices $T$ such that $T_{1}^{3}=T_{2}^{3}=0$ and $T_{1}^{1} T_{2}^{2}-$ $T_{2}^{1} T_{1}^{2}=\left(T_{3}^{3}\right)^{2}$

Deforming this algebra, we consider the quantum space obtained when relations (1) are replaced by

$$
\left(R_{x x}\right)\left\{\begin{aligned}
x^{1} x^{2}-q x^{2} x^{1}-s\left(x^{3}\right)^{2} & =0 \\
x^{1} x^{3}-u x^{3} x^{1} & =0 \\
x^{2} x^{3}-u^{-1} x^{3} x^{2} & =0
\end{aligned}\right.
$$

Let $V$ be the vector space of column vectors $X$ with elements $x^{i}$. The matrix $T$ now has non commuting elements and acts on $X$ according to the definition [5] :

$$
\delta: X \longrightarrow \delta(X)=T \otimes X
$$

Relations $R_{x x}$ defined by (2) will be preserved by this action provided the following 
commutation relations hold:

$$
\begin{array}{cc}
T_{1}^{1} T_{3}^{3}=T_{3}^{3} T_{1}^{1} & T_{1}^{2} T_{3}^{3}=u^{-2} T_{3}^{3} T_{1}^{2} \\
T_{2}^{1} T_{3}^{3}=u^{2} T_{3}^{3} T_{2}^{1} & T_{2}^{2} T_{3}^{3}=T_{3}^{3} T_{2}^{2} \\
T_{3}^{1} T_{3}^{3}=u T_{3}^{3} T_{3}^{1} & T_{3}^{2} T_{3}^{3}=u^{-1} T_{3}^{3} T_{3}^{2} \\
T_{1}^{1} T_{1}^{2}=q T_{1}^{2} T_{1}^{1} & T_{2}^{1} T_{2}^{2}=q T_{2}^{2} T_{2}^{1} \\
u T_{1}^{1} T_{3}^{2}-q T_{3}^{2} T_{1}^{1}=q u T_{1}^{2} T_{3}^{1}-T_{3}^{1} T_{1}^{2} \\
T_{2}^{1} T_{3}^{2}-q u T_{3}^{2} T_{2}^{1}=q T_{2}^{2} T_{3}^{1}-u T_{3}^{1} T_{2}^{2} \\
T_{1}^{1} T_{2}^{2}-T_{2}^{2} T_{1}^{1}=q T_{1}^{2} T_{2}^{1}-q^{-1} T_{2}^{1} T_{1}^{2} \\
\left(T_{1}^{1} T_{2}^{2}-q T_{1}^{2} T_{2}^{1}\right) s=s\left(T_{3}^{3}\right)^{2}-T_{3}^{1} T_{3}^{2}+q T_{3}^{2} T_{3}^{1}
\end{array}
$$

In that case, the action $\delta$ becomes defined as a mapping of $\mathbb{C}<x>/ R_{x x}$ onto itself.

\section{$3 \quad R$-matrix and invariant differential calculus}

In the present case, a differential calculus on $\mathbb{C}\langle x\rangle / R_{x x}$ that is invariant under the action of $T$ can be constructed if there exists a matrix $\widehat{R}$ with the following properties [0] :

- $\widehat{R}$ is defined by the relations

$$
\widehat{R}_{k l}^{j i} T_{m}^{k} T_{n}^{l}=T_{l}^{j} T_{k}^{i} \widehat{R}_{m n}^{l k}
$$

- $\widehat{R}$ has two eigenspaces, $V_{1}$ and $V_{2}$ that can be identified with the variables and the one-forms quantum spaces respectively. Space $V_{1}$ has dimension six and is determined by the relations $\left(R_{x x}\right)$ given in (2) . 
The determination of $\widehat{R}$ is performed by assuming that relations (44 8 ) can be cast into the form (9) and solving the corresponding equations. In addition, we impose two natural requirements:

(i) The determinant of $T$ is different from zero so that the set of matrices $T$ can be made into a Hopf algebra.

(ii) The ordering of monomials such as $T_{j}^{i} T_{l}^{k} T_{n}^{m}$ is independent of the procedure used, when the associativity of the algebra $C<T>$ is taken into account.

With these conditions, it is found that a $\widehat{R}$-matrix exists only if $q=u^{2}$ and it is given by:

$$
\widehat{R}=\left(\begin{array}{ccccccccc}
1 & 0 & 0 & 0 & 0 & 0 & 0 & 0 & 0 \\
0 & 0 & 0 & u^{2} & 0 & 0 & 0 & 0 & s \\
0 & 0 & 0 & 0 & 0 & 0 & u & 0 & 0 \\
0 & u^{-2} & 0 & 0 & 0 & 0 & 0 & 0 & -s / u^{2} \\
0 & 0 & 0 & 0 & 1 & 0 & 0 & 0 & 0 \\
0 & 0 & 0 & 0 & 0 & 0 & 0 & 1 / u & 0 \\
0 & 0 & 1 / u & 0 & 0 & 0 & 0 & 0 & 0 \\
0 & 0 & 0 & 0 & 0 & u & 0 & 0 & 0 \\
0 & 0 & 0 & 0 & 0 & 0 & 0 & 0 & 1
\end{array}\right)
$$

It can be shown that this matrix $\widehat{R}$ is equal to its inverse and verifies the Yang-Baxter equation:

$$
(\widehat{R} \otimes 1)(1 \otimes \widehat{R})(\widehat{R} \otimes 1)=(1 \otimes \widehat{R})(\widehat{R} \otimes 1)(1 \otimes \widehat{R})
$$


where 1 is the unit matrix of $G L(3)$. The matrix $\widehat{R}$ has two eigenspaces that correspond to the variables quantum space defined by the relations $R_{x x}$ and to the one-forms quantum space defined by :

$$
R_{\xi \xi}: \begin{cases}\left(\xi^{1}\right)^{2}=0, & \left(\xi^{2}\right)^{2}=0 \\ \left(\xi^{3}\right)^{2}=0, & \xi^{2} \xi^{1}=-u^{-2} \xi^{1} \xi^{2} \\ \xi^{1} \xi^{3}=-u \xi^{3} \xi^{1}, & \xi^{2} \xi^{3}=-u^{-1} \xi^{3} \xi^{2}\end{cases}
$$

Result: If the existence of a $\widehat{R}$-matrix is assumed and if conditions (i) and (ii) are satisfied, then an invariant differential calculus can be set up on $C<x>/ R_{x x}$ if and only if $q=u^{2}$.

In the next section, the same result is obtained without assuming the existence of a $\widehat{R}$-matrix.

\section{Invariant exterior algebra}

Consider a vector $\Xi \in V$ with components $\xi^{i}, i=1,2,3$ and form $V \otimes V$. The space of invariant forms can be constructed directly as an invariant subspace $V_{2}$ of $V \otimes V$ that is supposed to be of dimension three or less. It will be done by finding the possible invariant relations between the $\xi^{i} \xi^{j}$.

An important tool for this derivation is the introduction of a degree $d^{\circ}$ on $C<T>$, i.e. of a homomorphism from $C<T>$ into $Z$. Explicitly, the degree operation $d^{\circ}$ associates with each element $T_{j}^{i}$ the power of $u$ present in the commutation relations (丰) of that element with $T_{3}^{3}$. Assuming that $u \neq 1$, we find the degrees of all the 
elements of $T$ :

$$
\begin{aligned}
& d^{\circ}\left(T_{1}^{1}\right)=d^{\circ}\left(T_{2}^{2}\right)=d^{\circ}\left(T_{3}^{3}\right)=0 \\
& d^{\circ}\left(T_{2}^{1}\right)=2 \\
& d^{\circ}\left(T_{1}^{2}\right)=-2 \\
& d^{\circ}\left(T_{3}^{1}\right)=1 \\
& d^{\circ}\left(T_{3}^{2}\right)=-1
\end{aligned}
$$

The degrees of all the monomials in $C<T>$ can then be deduced.

Consider a quadratic relation $R_{1}=0$ between the $\xi$. After action of the homomorphism $\delta \otimes \delta$ defined in (3), a new relation $(T \otimes T) \otimes R_{1}=0$ is obtained which contains monomials $T_{j}^{i} T_{l}^{k}$ of known degrees. Invariance of relation $R_{1}=0$ then implies that terms of different degrees vanish separately. Applying this remark systematically allows to find all possible invariant relations as will now be sketched.

Let $\xi^{\prime i} \xi^{\prime j}$ denote the components of the vector $\Xi^{\prime} \otimes \Xi^{\prime} \equiv(T \otimes T) \otimes(\Xi \otimes \Xi)$. The monomial $\left(\xi^{\prime 1}\right)^{2}$ is the only one containing a term of degree 4 . Hence it cannot be involved in any quadratic relation except $\left(\xi^{1}\right)^{2}=0$. The same reasoning with $\left(\xi^{\prime 2}\right)^{2}$ and degree $(-4)$ leads to $\left(\xi^{2}\right)^{2}=0$. In the remaining transformed relations, terms of degree $(+2)(\operatorname{resp}(-2))$ come from monomials $\xi^{\prime 1} \xi^{\prime 3}$ and $\xi^{\prime 3} \xi^{\prime 1}\left(\operatorname{resp} \xi^{\prime 2} \xi^{\prime 3}\right.$ and $\left.\xi^{\prime 3} \xi^{\prime 2}\right)$. This implies that $\xi^{1} \xi^{3}$ and $\xi^{2} \xi^{3}$ are independent monomials. To generate $V_{2}$, we need only a third independent monomial which can be chosen as $\xi^{1} \xi^{2}, \xi^{2} \xi^{1}$ or $\left(\xi^{3}\right)^{2}$. Choosing 
$\xi^{1} \xi^{2}$, we can write the general form of the invariant relations as:

$$
\begin{aligned}
& \left(\xi^{1}\right)^{2}=0 \\
& \left(\xi^{2}\right)^{2}=0 \\
& \left(\xi^{3}\right)^{2}=k \xi^{1} \xi^{2} \\
& \xi^{2} \xi^{1}=\xi \xi^{1} \xi^{2} \\
& \xi^{3} \xi^{1}=\lambda \xi^{1} \xi^{3}+\lambda_{12} \xi^{1} \xi^{2} \\
& \xi^{3} \xi^{2}=\mu \xi^{2} \xi^{3}+\mu_{12} \xi^{1} \xi^{2}
\end{aligned}
$$

The conditions of invariance of these commutation relations yield new constraints on the elements of matrix $T$ which are consistent provided that $k=\lambda_{12}=\mu_{12}=0$.

If instead we choose $\left(\xi^{3}\right)^{2}$ as third independent monomial, we obtain a different situation only if $\xi^{1} \xi^{2}=0$. In that case $\xi^{1} \xi^{\prime 2}$ contains only one term of degree 0 , namely $T_{3}^{1} T_{3}^{2}\left(\xi^{3}\right)^{2}$ that cannot vanish. Hence this case is impossible.

We prove in the same way that the dimension of $V_{2}$ cannot be equal to 2 .

Result: If we assume that the differentials are related by six (or more) relations, the only possible set of relations is given by (14) with $k=\lambda_{12}=\mu_{12}=0$. These relations will be denoted by $R_{\xi \xi}^{\prime}$.

The constraint of invariance of $R_{\xi \xi}^{\prime}$ has been explicitly studied in [6]. The consistency requirement for the ordering of terms containing a product of three $T_{j}^{i}$ has led to the condition $q=u^{2}$. As a consequence, the relations $R_{\xi \xi}^{\prime}$ become identical with $R_{\xi \xi}$ given in (12). 


\section{Quantum groups}

\subsection{A quantum group with seven generators}

A unique set of relations $\left(R_{x x}, R_{\xi, \xi}\right)$ has been obtained. They are given by (2, 12) with $q=u^{2}$ and define the $x$ and $\xi$ spaces respectively. The constraints of invariance by homomorphism $\delta$ and consistency of the computations lead to the following relations $R_{T T}$ between the elements $T_{j}^{i}$ :

$$
\begin{array}{llll}
T_{1}^{1} T_{2}^{1}=u^{-2} T_{2}^{1} T_{1}^{1} & T_{1}^{1} T_{3}^{1}=u^{-1} T_{3}^{1} T_{1}^{1} & T_{1}^{1} T_{2}^{2}=T_{2}^{2} T_{1}^{1} & T_{1}^{1} T_{1}^{2}=u^{2} T_{1}^{2} T_{1}^{1} \\
T_{1}^{1} T_{3}^{2}=u T_{3}^{2} T_{1}^{1} & T_{1}^{1} T_{3}^{3}=T_{3}^{3} T_{1}^{1} & T_{2}^{1} T_{3}^{1}=u T_{3}^{1} T_{2}^{1} & T_{2}^{1} T_{2}^{2}=u^{2} T_{2}^{2} T_{2}^{1} \\
T_{2}^{1} T_{1}^{2}=u^{4} T_{1}^{2} T_{2}^{1} & T_{2}^{1} T_{3}^{2}=u^{3} T_{3}^{2} T_{2}^{1} & T_{2}^{1} T_{3}^{3}=u^{2} T_{3}^{3} T_{2}^{1} & T_{3}^{1} T_{2}^{2}=u T_{2}^{2} T_{3}^{1} \\
T_{3}^{1} T_{1}^{2}=u^{3} T_{1}^{2} T_{3}^{1} & T_{3}^{1} T_{3}^{3}=u T_{3}^{3} T_{3}^{1} & T_{2}^{2} T_{1}^{2}=u^{2} T_{1}^{2} T_{2}^{2} & T_{2}^{2} T_{3}^{2}=u T_{3}^{2} T_{2}^{2} \\
T_{2}^{2} T_{3}^{3}=T_{3}^{3} T_{2}^{2} & T_{1}^{2} T_{3}^{2}=u^{-1} T_{3}^{2} T_{1}^{2} & T_{1}^{2} T_{3}^{3}=u^{-2} T_{3}^{3} T_{1}^{2} & T_{3}^{2} T_{3}^{3}=u^{-1} T_{3}^{3} T_{3}^{2} \\
T_{3}^{1} T_{3}^{2}=u^{2} T_{3}^{2} T_{3}^{1}+s\left(T_{1}^{1} T_{2}^{2}-u^{2} T_{1}^{2} T_{2}^{1}-\left(T_{3}^{3}\right)^{2}\right)=0 &
\end{array}
$$

The inverse matrix can now be completely determined and is given by :

$$
T^{-1}=\left(\begin{array}{ccc}
T_{2}^{2} T_{3}^{3} & -u^{2} T_{2}^{1} T_{3}^{3} & T_{2}^{1} T_{3}^{2}-u T_{3}^{1} T_{2}^{2} \\
-u^{-2} T_{1}^{2} T_{3}^{3} & T_{1}^{1} T_{3}^{3} & -u^{-2} T_{1}^{1} T_{3}^{2}+u^{-3} T_{3}^{1} T_{1}^{2} \\
0 & 0 & T_{1}^{1} T_{2}^{2}-u^{-2} T_{2}^{1} T_{1}^{2}
\end{array}\right) \times D^{-1}
$$

where the determinant $D$ can be written as :

$$
D \equiv\left(T_{1}^{1} T_{2}^{2}-u^{-2} T_{2}^{1} T_{1}^{2}\right) T_{3}^{3}
$$

Remark that the determinant is not central and its inverse $D^{-1}$ must be added to the set of generators of the algebra. Its commutation relations $R_{T D^{-1}}$ are easily deduced 
from those of $D$ and read:

$$
\begin{array}{lll}
D^{-1} T_{1}^{1}=T_{1}^{1} D^{-1} & u^{-6} D^{-1} T_{2}^{1}=T_{2}^{1} D^{-1} & u^{-3} D^{-1} T_{3}^{1}=T_{3}^{1} D^{-1} \\
D^{-1} T_{2}^{2}=T_{2}^{2} D^{-1} & D^{-1} T_{1}^{2}=u^{-6} T_{1}^{2} D^{-1} & D^{-1} T_{3}^{2}=u^{-3} T_{3}^{2} D^{-1} \\
D^{-1} T_{3}^{3}=T_{3}^{3} D^{-1} & &
\end{array}
$$

With these definitions, it may be verified that $H_{8} \equiv C<T, D^{-1}>/ R_{T T} \cup R_{T D^{-1}}$ is a Hopf algebra with co-product $\Delta$, co-unit $\epsilon$ and antipode $S$ defined by :

$$
\begin{array}{r}
\Delta(T) \equiv T \otimes T, \quad \Delta\left(D^{-1}\right) \equiv D^{-1} \otimes D^{-1} \\
\epsilon\left(T, D^{-1}\right) \equiv(I, 1), \quad S(T) \equiv T^{-1}, \quad S(D) \equiv D^{-1}
\end{array}
$$

Now we can apply the usual method ([3], [4] ) to obtain the quadratic relations between the variables, the differentials and the derivatives [6]. They are given by:

$$
\begin{aligned}
x^{k} \xi^{l} & =\widehat{R}_{m n}^{k l} \xi^{m} x^{n} \\
\partial_{k} \xi^{l} & =\widehat{R}_{k n}^{-1 l m} \xi^{n} \partial_{m} \\
\partial_{l} x^{k} & =\delta_{l}^{k}+\widehat{R}_{l n}^{k m} x^{n} \partial_{m}
\end{aligned}
$$

\subsection{A quantum group with nine generators}

Once the matrix $\widehat{R}$ has been explicitly computed, it is possible to introduce a new quantum matrix $t$ with nine elements satisfying the relations $R_{t t}$ deduced from (9):

$$
\widehat{R}_{k l}^{j i} t_{m}^{k} t_{n}^{l}=t_{l}^{j} t_{k}^{i} \widehat{R}_{m n}^{l k}
$$


The computation of the inverse $t^{-1}$ yields:

$$
\left(\begin{array}{ccc}
t_{2}^{2} t_{3}^{3}-u t_{3}^{2} t_{2}^{3} & -u^{2} t_{2}^{1} t_{3}^{3}+u^{3} t_{3}^{1} t_{2}^{3} & t_{2}^{1} t_{3}^{2}-u t_{3}^{1} t_{2}^{2} \\
-u^{-2} t_{1}^{2} t_{3}^{3}+u^{-3} t_{3}^{2} t_{1}^{3} & t_{1}^{1} t_{3}^{3}-u^{-1} t_{3}^{1} t_{1}^{3} & -u^{-2} t_{1}^{1} t_{3}^{2}+u^{-3} t_{3}^{1} t_{1}^{2} \\
t_{1}^{2} t_{2}^{3}-u^{-2} t_{2}^{2} t_{1}^{3} & -u^{2} t_{1}^{1} t_{2}^{3}+t_{2}^{1} t_{1}^{3} & t_{1}^{1} t_{2}^{2}-u^{-2} t_{2}^{1} t_{1}^{2}
\end{array}\right) d^{-1}
$$

with the determinant $d$ of $t$ equal to:

$$
d=t_{1}^{1} t_{2}^{2} t_{3}^{3}+t_{3}^{1} t_{1}^{2} t_{2}^{3}+u^{-3} t_{2}^{1} t_{3}^{2} t_{1}^{3}-u^{-1} t_{1}^{1} t_{3}^{2} t_{2}^{3}-u^{-2} t_{2}^{1} t_{1}^{2} t_{3}^{3}-u^{-2} t_{3}^{1} t_{2}^{2} t_{1}^{3}
$$

It can be verified that $d$ is not a central element of $C<t>$ and therefore must be added to this algebra. The commutation relations $R_{t d^{-1}}$ of $d^{-1}$ with the generators $t_{j}^{i}$ are:

$$
\begin{aligned}
& t_{1}^{1} d^{-1}=d^{-1} t_{1}^{1}, \quad t_{2}^{1} d^{-1}=u^{-6} d^{-1} t_{2}^{1}, \quad t_{3}^{1} d^{-1}=u^{-3} d^{-1} t_{3}^{1}, \\
& t_{2}^{2} d^{-1}=d^{-1} t_{2}^{2}, \quad t_{1}^{2} d^{-1}=u^{4} d^{-1} t_{1}^{2}, \quad t_{3}^{2} d^{-1}=u^{-3} d^{-1} t_{3}^{2}, \\
& t_{1}^{3} d^{-1}=u^{3} d^{-1} t_{1}^{3}, \quad t_{2}^{3} d^{-1}=u^{-3} d^{-1} t_{2}^{3}, \quad t_{3}^{3} d^{-1}=d^{-1} t_{3}^{3} .
\end{aligned}
$$

In this manner, $H_{10} \equiv C<t, d^{-1}>/ R_{t t} \cup R_{t d^{-1}}$ is endowed with a structure of Hopf algebra.

Thus two Hopf algebras, $H_{8}$ and $H_{10}$, have been constructed. Both preserve the same differential calculus on the deformed oscillator algebra defined by $R_{x x}$ with $q=u^{2}$. In addition, the construction ensures that $H_{10}$ contains $H_{8}=C<T, D^{-1}>/ R_{T T} \cup$ $R_{T D^{-1}}$ as a Hopf subalgebra.

\section{Conclusion}

We have been able to deform simultaneously the Weyl-Heisenberg algebra and its group of invariance (a subgroup of $G L(3)$ ). In addition, an invariant differential calculus 
has been set up on the resulting quantum space. However, it must be stressed that the whole construction cannot be carried out for arbitrary values of the deformation parameters and that the final result depends only on one complex number $u$.

The constraint on the values of the parameters can be removed when the requirement of invariance by a seven-generator quantum group is lifted. A purely algebraic approach [4] can be developed and the commutation relations are then shown to be invariant by a quantum matrix belonging to a three-parameter deformation of $G L(3)$ [7] [8].

The construction performed in this paper has yielded two quantum groups and their associated Hopf algebras $H_{10}, H_{8}$, which have ten and eight generators respectively. These algebras are original deformations of $G L(3)$ and of its subgroup $G$. Moreover, the smaller one $H_{8}$ is embedded in $H_{10}$ as a true Hopf subalgebra. 


\section{References}

[1] Woronowicz, S.L.(1989) Differential Calculus on Compact Matrix Pseudogroups (Quantum Groups) Commun. Math. Phys., 122, pp. 125-170.

[2] Bernard, D. (1990) Quantum Lie Algebras and Differential Calculus on Quantum Groups Progress of Theoretical Physics Supplement 102 pp. 49-66.

[3] Wess, J. and Zumino, B. (1990) Covariant Differential Calculus on the Quantum Hyperplane Nucl. Phys. (Proc. Suppl.), B 18, pp. 301-313.

[4] Zumino, G. (1992) Differential calculus on quantum spaces and quantum groups, in Group Theoretical Methods in Physics, M.A. del Olmo, M.Santander and J.Mateos Guilarte (Eds.), Anales de Física Monografias, CIEMAT (Spain), pp. 41-59.

[5] Reshetikhin, N.Yu., Takhtadzhyan, L.A. and Faddeev, L.D. (1990) Quantization of Lie groups and Lie algebras, Leningrad Math. J., 1, pp. 193-225.

[6] Bertrand, J. and Irac-Astaud, M. (1995) Invariant differential calculus on a deformation of the Weyl-Heisenberg algebra, Modern Group Theoretical Methods in Physics, p. 37, Kluwer Academic Publishers.

[7] Irac-Astaud, M.(1996) Differential calculus on a three-parameter oscillator algebra, to be published in Reviews in Mathematical Physics

[8] Irac-Astaud, M.(1996) A three-parameter deformation of the Weyl-Heisenberg algebra : differential calculus and invariance, to be published in the Proceedings of 
the 5th international Colloquium "Quantum Groups and Integrable systems" of Prague, Czechoslovak Journal of Physics. 